\title{
Temporal, geographical and demographic trends of stroke prevalence in China: a systematic review and meta-analysis
}

\author{
Jinli Liu ${ }^{1}$, Zumin Shi ${ }^{2}$, Ruhai Bai ${ }^{1}$, Jinge Zheng ${ }^{1}$, Shuang Ma ${ }^{1}$, Junxiang Wei ${ }^{1}$, Guangzhi Liu ${ }^{3}$, \\ Youfa Wang ${ }^{1,4}$
}

${ }^{1}$ School of Public Health, Global Health Institute, Xi'an Jiaotong University Health Science Center, Xi'an, China; ${ }^{2}$ Human Nutrition Department, College of Health Sciences, QU Health, Qatar University, Doha, Qatar; ${ }^{3}$ Department of Neurology, Beijing Anzhen Hospital, Capital Medical University, Beijing, China; ${ }^{4}$ Fisher Institute of Health and Well-Being, Department of Nutrition and Health Sciences, College of Health, Ball State University, Muncie, Indiana, USA

Contributions: (I) Conception and design: Y Wang, Z Shi, J Liu; (II) Administrative support: Y Wang; (III) Provision of study materials or patients: R Bai, J Liu; (IV) Collection and assembly of data: J Liu, J Zheng, S Ma; (V) Data analysis and interpretation: Z Shi, Y Wang, J Liu; (VI) Manuscript writing: All authors; (VII) Final approval of manuscript: All authors.

Correspondence to: Youfa Wang, MD, PhD, MS, Professor \& Dean. Global Health Institute, School of Public Health, Xi'an Jiaotong University Health Science Center, Xi’an, China. Email: youfawang@gmail.com.

\begin{abstract}
Background: China has made large efforts to reduce stroke prevalence. We aimed to systematically examine the prevalence of stroke in China over the past two decades.

Methods: Databases, including China National Knowledge Infrastructure, Wanfang, VIP, and PubMed, were systematically searched for studies published in English or Chinese that reported stroke prevalence in China during 2000-2017. Meta-analysis was conducted to estimate the pooled stroke prevalence and the variations in stroke prevalence subgroups stratified by age, gender, time period, and region.

Results: In total, 96 papers met the inclusion criteria. Meta-analysis showed that the overall estimated national prevalence was $5.1 \%(5.0-5.3 \%)$ with large variations across regions: $3.1 \%(2.5-3.6 \%)$ in south China, 3.4\% (3.0-3.8\%) in southwest China, 3.6\% (3.3-3.8\%) in east China, $5.0 \%(4.7-5.4 \%)$ in central China, 5.8\% (4.6-7.1\%) in northwest China, 6.0\% (5.0-7.0\%) in northeast China, and 8.0\% (7.4-8.5\%) in north China. Men had a higher prevalence than women $[7.3 \%(6.9-7.7 \%)$ vs. 5.6\% (5.2-6.0\%)]. Stroke prevalence increased with age, was $1.2 \%(1.0-1.3 \%), 2.9 \%(2.6-3.2 \%), 5.9 \%(5.2-6.5 \%)$, and $8.7 \%(8.0-$ $9.5 \%)$ in the $40-49,50-59,60-69$, and $\geq 70$ years old groups, respectively.
\end{abstract}

Conclusions: Men, people being older, or living in northern China had higher stroke prevalence. More vigorous efforts are needed in China to prevent stroke.

Keywords: Stroke; trends; meta-analysis; China

Submitted Dec 13, 2019. Accepted for publication Jul 27, 2020.

doi: 10.21037/atm-19-4342

View this article at: http://dx.doi.org/10.21037/atm-19-4342

\section{Introduction}

Stroke is the second leading cause of death worldwide and in China, where a fifth of the world's population resides. With over 2 million new cases annually, stroke is associated with the highest disability-adjusted life-years lost among all chronic diseases in China (1-3). The burden of stroke has increased worldwide because of shifts in people's lifestyles and population aging, especially in low- and middle-income countries (4). Stroke also creates tremendous economic pressure on health care systems $(5,6)$. The annual incidence of stroke is expected to increase to over 23 million and annual deaths to 7.8 million by 2030 in the absence of effective global public health responses $(7,8)$.

In China, about 2.4 million people have a new onset of stroke each year. About $75 \%$ of individuals living with 
stroke will develop neurological sequela that leads to various degrees of disability $(9,10)$. Stroke also brings a heavy financial burden to the affected families and society. Over the past decade, China has implemented several national and regional programs for stroke prevention and control. These included the well-known Healthy China 2020 and Healthy China 2030 programs that were launched in 2012 and 2016, respectively. Regional reports show the beneficial effects of these programs, with a risk of stroke in high-risk individuals and the general population possibly benefitting more than others from such programs (11). A systematic examination of the trends of stroke prevalence in China will enable us to gain important insights into the effectiveness of these intervention programs.

This study aimed to systematically examine the temporal changes in the prevalence of stroke and also differences in the prevalence stratified by age, gender, and region over the past two decades in China. This information will aid intervention efforts in future. We present the following article in accordance with the PRISMA reporting checklist (available at http://dx.doi.org/10.21037/atm-19-4342) (12).

\section{Methods}

\section{Literature search}

We searched the following databases: (I) the China National Knowledge Infrastructure (CNKI) (http://www.cnki.net); (II) Wanfang Database (http://www.wanfangdata.com.cn/index. html); (III) China Science and Technology Journal Database (VIP) Database (http://www.cqvip.com/); and (IV) PubMed (https://www.ncbi.nlm.nih.gov/pmc/). We searched for studies published between January 1, 2000, and December 31, 2017, using the search terms "stroke" OR "transient ischemic attack" OR "intracerebral hemorrhage" OR "isch(a) emic stroke" OR" intraparenchymal" OR "subarachnoid" OR "h(a)emorrhage" AND "Chinese" OR "China" AND "prevalence" OR "epidemic." We also reviewed the reference lists of the retrieved publications to identify any additional relevant studies. Our meta-analysis was conducted according to the preferred reporting items of the Meta-Analysis of Observational Studies in Epidemiology (MOOSE) statement for meta-analyses of observational studies (13).

\section{Study selection}

Studies were included if they met the following criteria: (I) the study population was Chinese; (II) the study population was aged 40 and over; (III) participants were selected from the general population or those who undertook routine physical examinations organized by employers; (IV) the main outcome was the prevalence of stroke; (V) a crosssectional study or large cohort study for monitoring purposes; (VI) sample size $>1,000$; and (VII) age-specific data were presented.

Studies were excluded when meeting the following criteria: (I) no age-specific data were provided and a single age group over 30 years; (II) the number of participants was not provided or the confidence interval could not be estimated; (III) the papers were published repeatedly; or (IV) participants were hospitalized patients or patients with certain diseases or other special groups (such as pregnant females, nursing mothers, smoking, drinking, military police, athletes, and those on weight loss or diet programs).

Our initial screening resulted in 3,016, 3,033, 2,272, and 1,577 articles from the CNKI, Wanfang, VIP, and PubMed databases, respectively. Ninety-six studies met the inclusion criteria (Figure 1). Two authors separately reviewed the studies. When there were differences between their assessments, a third author provided an additional assessment.

\section{Data extraction}

We extracted prevalence data of cross-sectional studies and cohort studies in the general population using a standardized data-collection form that included the following information: authors, publication year, study design, study aim/objective, study period, region, sample sizes, stroke prevalence (\%) and $95 \%$ confidence interval (CI). Seven geographic regions in China were defined as north (Beijing, Tianjin, Hebei, Shanxi, and Inner Mongolia); northeast (Liaoning, Jilin, and Heilongjiang); northwest (Ningxia, Xinjiang, Qinghai, Shaanxi, and Gansu); central (Hubei, Hunan, and Henan); east (Shandong, Jiangsu, Anhui, Zhejiang, Fujian, Jiangxi, and Shanghai); southwest (Sichuan, Yunnan, Guizhou, Tibet, and Chongqing); south (Guangdong, Guangxi, and Hainan); special administrative region (Hong Kong and Macau); and Taiwan.

\section{Quality of literature evaluation}

For evaluation of the included studies, we used the literature quality assessment criteria proposed by Suriah AR, Chong TJ and Yeoh BY (Table S1) (14). Based on these quality assessment criteria, studies were scored 


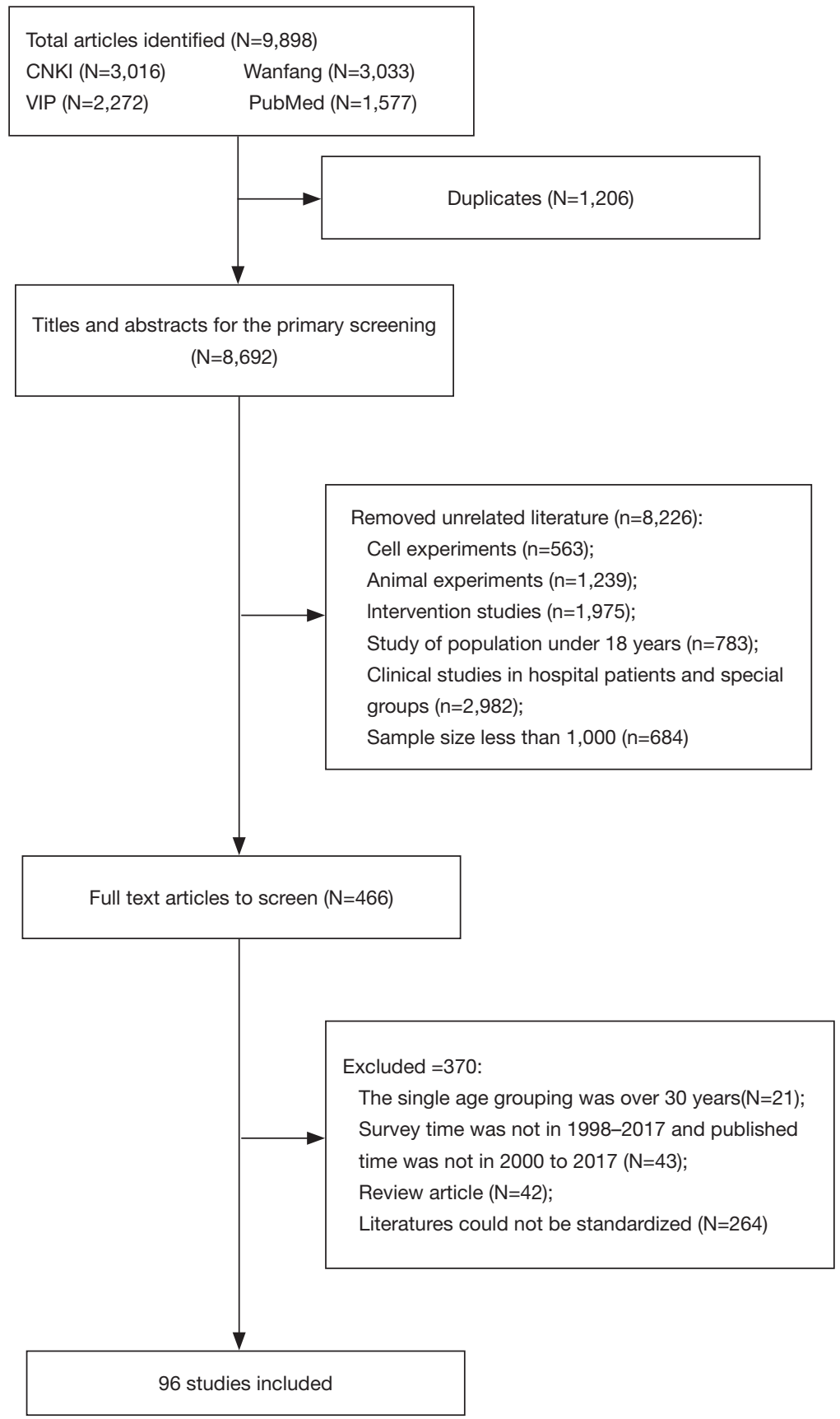

Figure 1 Flowchart of systematic review procedure to select the 96 studies included.

from 1 to 5 (highest to lowest quality score, respectively). A score of $1=$ nationally representative studies with large sample sizes and that employed random selection techniques; a score of $2=$ large, randomly selected samples from an entire state in the country; a score of $3=$ employed random selection techniques within a specified number of sampling units (e.g., two towns, three clinics); a score of $4=$ included large sample sizes $(>1,000$ persons) even though the samples were not randomly selected; and a score of $5=$ small, non-randomly selected samples (14). The literature research yielded 96 eligible studies (Table S2). Of these, 29 studies reported gender- 
specific prevalence. Six were national surveys (Table S3). In general, the studies reviewed in this meta-analysis were of good quality: 36 studies scored 1, 48 studies scored 2, 1 study scored 3, 11 studies scored 4. and 0 study scored 5 . We conducted a subgroup analysis of stroke prevalence among the five criteria used to evaluate the paper. The results showed no statistical significance $(\mathrm{P}=0.093)$.

\section{Statistical analysis}

The prevalence of stroke and its $95 \%$ CI were estimated using STATA/SE (Version 15.0; StataCorp LLC, College Station, TX, USA). The overall prevalence and age-, gender-, period-, and region-specific prevalence were estimated by meta-analysis, based on a random effects model. We estimated the pooled prevalence for each of the seven regions across China, and we divided the studies into the three periods of 2000-2005, 2006-2011, and 2012-2017 based on the published dates. If the time or age spans covered different periods or age groups, we calculated the average value for the corresponding periods or age groups. We used the sixth Chinese census data in 2010 to calculate the age-standardized prevalence, and a linear regression model was conducted for trend tests for age and periods. Statistical significance was set at $\mathrm{P}<0.05$.

\section{Results}

\section{Overall stroke prevalence in China}

We included a total of 96 studies that documented 169,958 stroke cases in 3,332,502 participants. The overall estimate of the national prevalence of stroke was $5.1 \%$ (95\% CI: $5.0-5.3 \%)$. In particular, the pooled stroke prevalence rates were $5.2 \%(4.8-5.6 \%)$ in $2000-2005,4.7 \%(4.5-4.9 \%)$ in 2006-2011, and 5.4\% (5.1-5.8\%) in 2012-2017 (Table 1, Figure S1A).

\section{Prevalence stratified by geographical regions}

The prevalence varied across different regions, with $8.0 \%$ (7.4-8.5\%) for north China, 6.0\% (5.0-7.0\%) for northeast China, $5.8 \%$ (4.6-7.1\%) for northwest China, 5.0\% (4.7$5.4 \%)$ for central China, $3.6 \%(3.3-3.8 \%)$ for east China, $3.4 \%(3.0-3.8 \%)$ for southwest China, 3.1\% (2.5-3.6\%) for south China, and $2.7 \%$ (2.3-3.0\%) for Taiwan (Table 1). Stroke prevalence was the highest in the north and the lowest in the south of China (Figure 2).
At the provincial level, the prevalence ranged from the highest $(8.9 \%)$ in Inner Mongolia to the lowest $(0.6 \%)$ in Tibet. Within north China, the prevalence of stroke was high in Beijing (8.3\%) and Inner Mongolia (8.9\%), but low in Hebei $(3.7 \%)$. The prevalence of stroke was below $5 \%$ in most of the provinces in south China, including $3.7 \%$ in Guangdong, 1.5\% in Guangxi, and 2.7\% in Taiwan.

\section{Prevalence stratified by age group}

The prevalence of stroke increased rapidly with age (Figure $3 A$ ), with prevalence rates of $1.2 \%(1.0-1.3 \%), 2.9 \%$ $(2.6-3.2 \%), 5.9 \%(5.2-6.5 \%)$, and $8.7 \%(8.0-9.5 \%)$ in the $40-49,50-59,60-69$, and $\geq 70$ age groups, respectively (Table 1). A significant increase in the prevalence of stroke was observed among those younger than 60 . The prevalence of stroke among those aged $40-49$ years doubled from $0.8 \%$ (0.5-1.0\%) in 2000-2005 to $1.5 \%(1.2-1.8 \%)$ in 2012-2017. In comparison, the prevalence among those aged 50-59 had increased 1.7 times, from $2.0 \%(1.5-2.6 \%)$ in $2000-2005$ to $3.4 \%(2.8-4.0 \%)$ in 2012-2017 (Table 2). However, during the same period, the prevalence decreased slightly among those aged older than 60 (Table 2).

\section{Prevalence stratified by gender}

Based on 29 gender-specific studies, that included 465,276 men (33,965 cases) and 335,708 women (18,800 cases), our analysis demonstrated that men $[7.3 \%(6.9-7.7 \%)]$ had a higher prevalence of stroke than women [5.6\% (5.2-6.0\%)] (Figure 3B). When stratified by the time period, the pooled prevalence in men increased from $7.1 \%(6.0-8.3 \%)$ in 2000 2005 to $7.3 \%(6.6-8.0 \%)$ in $2006-2011$, then to $7.5 \%(6.7-$ $8.2 \%)$ in $2012-2017$, whereas the corresponding prevalence in women were $5.4 \%(4.5-6.4 \%), 6.2 \%(5.5-6.8 \%)$, and $5.2 \%(4.5-5.8 \%)$ (Table 1). When stratified by age groups, the prevalence rates in men were $1.2 \%(0.9-1.6 \%), 4.2 \%$ (3.3-5.1\%), 7.8\% (6.5-9.2\%), and $12.0 \%(10.0-13.9 \%)$ in the age groups of 40-49, 50-59, 60-69, and $\geq 70$, respectively. For women, the corresponding rates were $0.9 \%(0.6-1.1 \%)$, $2.7 \%(2.1-3.3 \%), 5.9 \%(4.8-7.0 \%)$, and $9.4 \%(7.9-11.0 \%)$, respectively (Table 1). The overall prevalence of stroke in men was higher than that in women throughout all time periods (Figure S1B) in all different age groups (Figure 3C).

\section{Discussion}

We systematically examined the prevalence of stroke in 
Table 1 Pooled stroke prevalence (\%) by age, time and region in China, based on meta-analysis ${ }^{\mathrm{a}}$

\begin{tabular}{|c|c|c|c|c|c|c|}
\hline Variable & \multicolumn{2}{|c|}{ All } & \multicolumn{2}{|c|}{ Male } & \multicolumn{2}{|c|}{ Female } \\
\hline \multicolumn{7}{|l|}{ Age (years) } \\
\hline $40-49$ & 1.2 & $1.0-1.3$ & 1.2 & $0.9-1.6$ & 0.9 & $0.6-1.1$ \\
\hline $50-59$ & 2.9 & $2.6-3.2$ & 4.2 & $3.3-5.1$ & 2.7 & $2.1-3.3$ \\
\hline$\geq 70$ & 8.7 & $8.0-9.5$ & 12.0 & $10.0-13.9$ & 9.4 & $7.9-11.0$ \\
\hline $\mathrm{F}_{\text {trend (age) }}$ & \multicolumn{2}{|c|}{169.035} & \multicolumn{2}{|c|}{57.829} & \multicolumn{2}{|c|}{35.877} \\
\hline$P_{\text {trend }}$ value & \multicolumn{2}{|c|}{$<0.001$} & \multicolumn{2}{|c|}{$<0.001$} & \multicolumn{2}{|c|}{$<0.001$} \\
\hline \multicolumn{7}{|l|}{ Time/year } \\
\hline 2012-2017 & 5.4 & $5.1-5.8$ & 7.5 & $6.7-8.2$ & 5.2 & $4.5-5.8$ \\
\hline $\mathrm{F}_{\text {trend (time) }}$ & \multicolumn{2}{|c|}{0.123} & \multicolumn{2}{|c|}{0.004} & \multicolumn{2}{|c|}{0.113} \\
\hline$P_{\text {trend }}$ value & \multicolumn{2}{|c|}{0.726} & \multicolumn{2}{|c|}{0.950} & \multicolumn{2}{|c|}{0.737} \\
\hline \multicolumn{7}{|l|}{ Region } \\
\hline North China & 8.0 & $7.4-8.5$ & 12.0 & $11.0-14.0$ & 9.4 & $8.2-11.0$ \\
\hline Northeast China & 6.0 & $5.0-7.0$ & 6.0 & $2.31-9.70$ & 5.7 & $1.6-9.7$ \\
\hline Northwest China & 5.8 & $4.6-7.1$ & - & - & - & - \\
\hline Central China & 5.0 & $4.7-5.4$ & 4.0 & $1.8-6.1$ & 3.3 & $1.2-5.4$ \\
\hline
\end{tabular}

${ }^{a}$, the total rate was calculated by meta-analysis after age was standardized according to the sixth Chinese census data in $2010 .-$, no date.

Chinese adults aged 40 and older based on studies published during 2000-2017. The overall prevalence of stroke was $5.1 \%$ in China. Men had a higher rate of prevalence than women consistently, and the prevalence increased significantly in recent years for those aged $40-59$, but it was relatively stable in those aged 60 and older. There were substantial regional differences in the rates of prevalence nationwide, with a high rate of prevalence in the north and a low one in the south.

The prevalence of stroke first decreased and then increased [5.2\% (4.8-5.6\%) in 2000-2005, 4.7\% (4.5-4.9\%) in 2006-2011, and 5.4\% (5.1-5.8\%) in 2012-2017] over the study period. However, the National Epidemiological Survey of Stroke in China highlighted a marked increase in stroke prevalence over the past decade (15). Due to data constraints, the temporal trend of stroke prevalence could not be analyzed annually. These may be subject to further investigation.

The stroke burden varied by regions. Consistent with previous findings (16), the prevalence of stroke in our study is higher in the north than in the south. Sun et al. showed that northeastern China had the highest incidence (441-486/100,000 person-years), whereas southern China had a significantly lower rate of incidence (81-136/100,000 person-years) (17). The difference is also in line with the difference of hypertension between north and south China. One study reported that the age-standardized prevalence of hypertension 


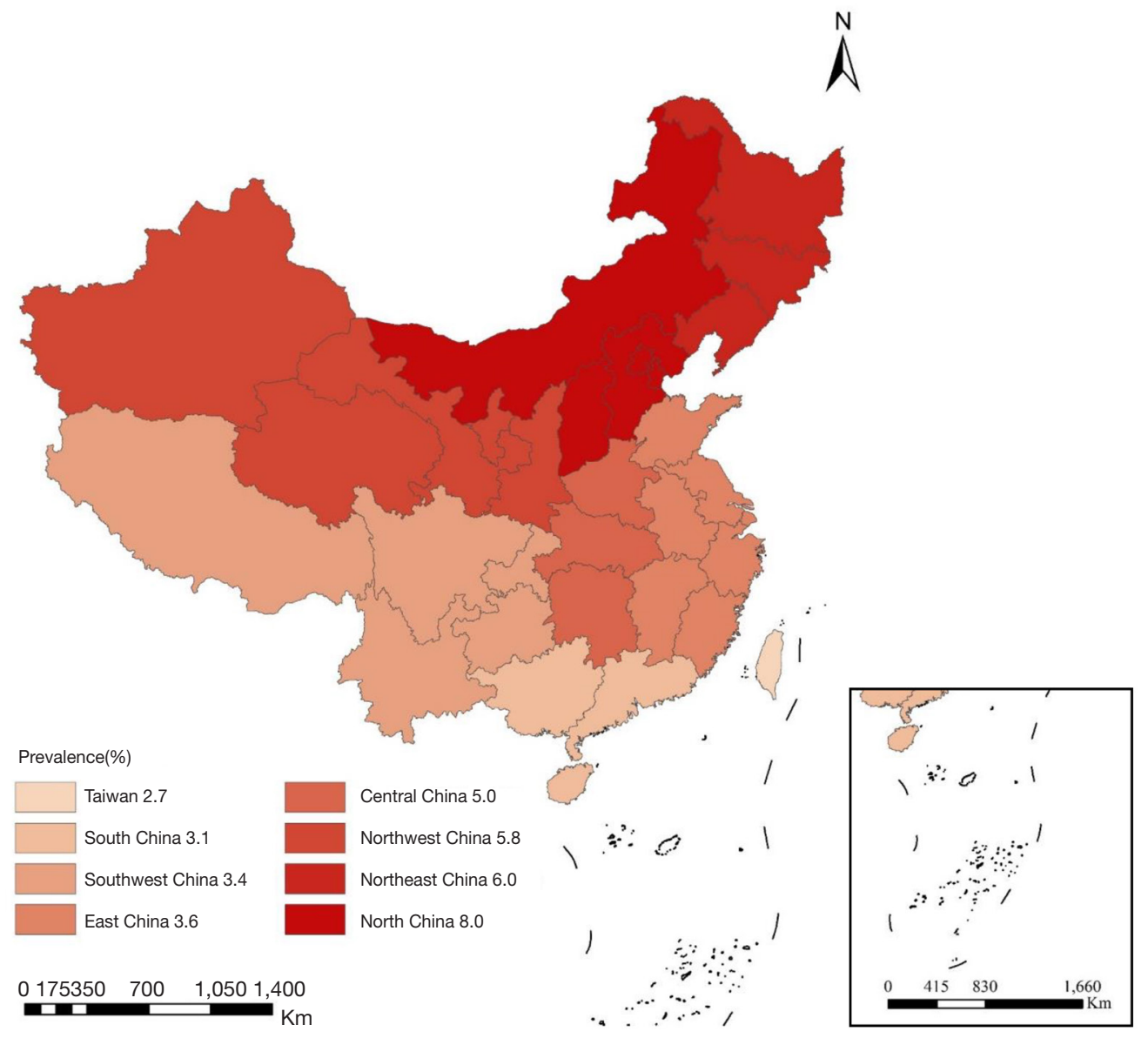

Figure 2 Regional differences in prevalence (\%) of stroke among adults aged $\geq 40$ years old in China during 2000-2017: based on metaanalysis of findings from 96 studies.

was significantly higher among residents living in the north than in the south of China $(33.8 \%$ vs. $23.3 \%)(18)$. Differences in diet, lifestyle factors, and climate may also contribute to the regional discrepancy in stroke (19-21). One of the main dietary differences between the south and the north is the staple food. In the south, rice is the main staple, while wheat is the staple in the north. Rice consumption seems beneficial for maintaining healthy body weight and blood pressure. In one study conducted in east China, the proportion of rice consumed as the main staple food was inversely related to hypertension risk (22).

The prevalence increased with age, which is consistent with other studies (23-25). Stroke has traditionally been regarded as a disease for the elderly (25). However, various studies have revealed that stroke has become increasingly common in young and middle-aged adults (40-59 years) in recent years (10). We reported a prevalence of rate of $1.5 \%$ among individuals aged 40-49 in 2012-2017. This may be due to two underlying reasons. First, in recent years, hypertension, a risk factor for stroke $(25,26)$, has become more prevalent among young and middle-aged adults (40-60 years) (27). Second, younger age groups have lower awareness and poorer management of stroke symptoms than older age groups (28).

The rapid increase of stroke rates among those aged 40-59 years old might be largely attributed to early life exposure to the Chinese famine in 1959-1961. The major modifiable risk factors for stroke were hypertension, diabetes mellitus, tobacco smoking, and hyperlipidemia, as well as lifestyle factors such as obesity, poor diet/nutrition, and physical inactivity (29). Studies have shown that people born during the Chinese famine have an increased risk of diabetes, obesity, and hypertension $(30,31)$. This may be due to the alterations in fetal nutrition and endocrine conditions 
A

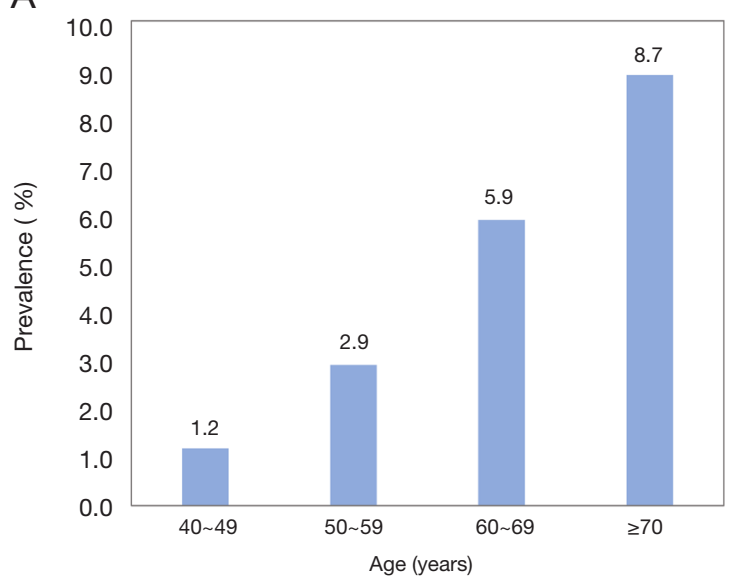

C

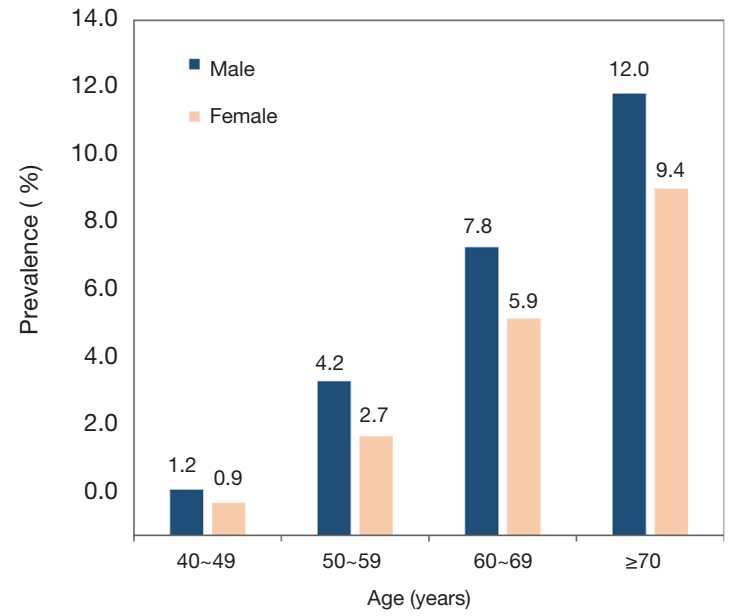

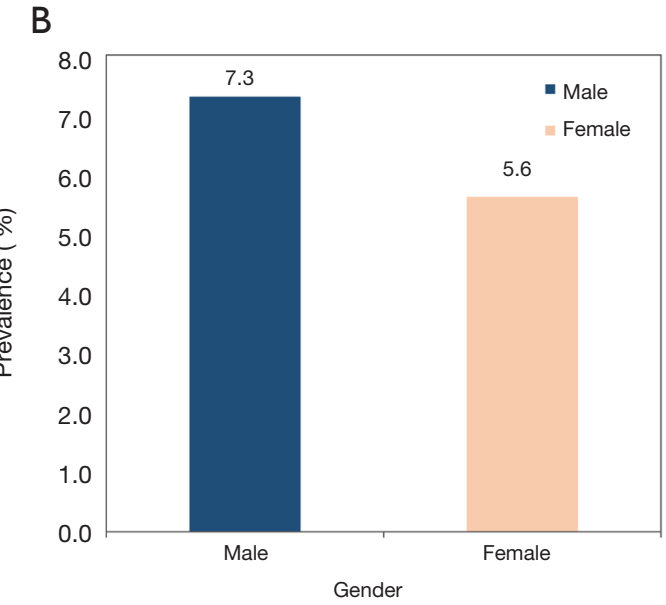

Figure 3 Meta-analysis of stroke prevalence (\%) by age group and gender in China (A) by age group (B) by gender (C) by age and gender.

leading to developmental adaptations, which permanently changes the structure of the body, causing them to develop body fat during intrauterine development, thereby making individuals prone to cardiovascular, metabolic and endocrine diseases. Furthermore, early life famine exposure exacerbates the association between hypertension and cardiovascular disease (CVD), including stroke (32). Using data from the China Health and Retirement Longitudinal Study (CHARLS), it has been reported that there was an increasing gradient of CVD risk associated with hypertension across late childhood, mid-childhood, and early childhood and fetal exposure to the Chinese famine. The odds ratio (OR) of hypertension for CVD was $1.69,2.35,2.48,3.35$, and 1.40 in late childhood, midchildhood, early childhood, fetal stages, and non-exposed cohorts, respectively (24). To what degree the early life famine exposure contributed to stroke has not yet been well studied. However, the early life famine affects hypertension and may affect stroke to some extent. One study revealed that early-life exposure to a Dutch famine might have affected lifestyle in adulthood (33). However, the traditional lifestyle factor intervention may not be effective in the group in the Chinese setting.

Stroke is more common among men than women $(34,35)$. Our study revealed that the prevalence of stroke in men is 1.3 times higher than that in women. This gender difference is consistent with other studies conducted in China and elsewhere (36). Many factors may explain this observation. First, the prevalence of smoking and alcohol consumption is much higher in men than women in China 
Table 2 Pooled stroke prevalence (\%) by age and period in China, based on meta-analysis

\begin{tabular}{|c|c|c|c|c|c|c|}
\hline Age (years) & \multicolumn{2}{|c|}{ 2000-2005 } & \multicolumn{2}{|c|}{ 2006-2011 } & \multicolumn{2}{|c|}{ 2012-2017 } \\
\hline $40-49$ & 0.8 & $0.5-1.0$ & 1.0 & $0.8-1.2$ & 1.5 & $1.2-1.8$ \\
\hline $50-59$ & 2.0 & $1.5-2.6$ & 2.8 & $2.3-3.2$ & 3.4 & $2.8-4.0$ \\
\hline $60-69$ & 6.4 & $5.0-7.9$ & 5.3 & $4.5-6.1$ & 6.2 & $5.3-7.2$ \\
\hline
\end{tabular}

Note: Based on the findings from 96 studies.

(62.4\% vs. 3.4\%, respectively, for smoking; and $33.0 \%$ vs. $2.0 \%$, respectively, for alcohol consumption) $(37,38)$. Smoking, an established and modifiable risk factor for stroke, accounts for $15-50 \%$ of all strokes, depending on age, gender, stroke subtype, and study population $(39,40)$. Second, there is a gender difference in the perception and management of weight, which is another major risk factor for stroke. Women are more likely to better manage their weight than men (41), and men have a significantly higher prevalence of being overweight or obese (BMI $\geq 24.0)$ than their female counterparts (42). In general, it is well-known that obesity plays a key role in health outcomes (43). Third, the different sex hormones between the genders (e.g., estrogen) may also contribute to the gender difference in stroke prevalence $(44,45)$.

This study has several main strengths: first, it summarizes the collective results from 96 studies. These studies covered large study samples, a wide range of geographic areas across China, and participants were more than 18 years old. Second, we conducted a meta-analysis of the findings and estimated stroke rates using random effect models. Third, we included six studies that used nationally representative data; some other studies included samples as large as 726,451 participants. Furthermore, some studies included all regions in mainland China: the 31 provinces, autonomous regions, and municipalities.

This study has some limitations. First, we tested the time trends, but the timeline of the study was divided into three periods and did not show trends in stroke prevalence by years. Second, although we included six studies that used nationally representative data, those studies that provided only regional data covered the 23 provinces and autonomous regions in China. Additionally, the included studies were of different quality: 36 studies scored 1, 48 studies scored 2, 1 study scored 3, 11 studies scored 4, and 0 study scored 5. Third, we aggregated the data by seven regions across
China, as only a limited number of studies provided data for each province. Collapsing the data by large regions may not present enough useful information for individual provinces in those regions. Finally, the 29 studies with gender-specific data provided a different prevalence estimate (higher) than the overall studies. Thus, our estimated overall stroke prevalence might be underestimated.

This study shows a large disease burden of stroke in China, with no sign of decline over the past two decades. With the population aging, China will face increasing challenges from chronic diseases, including stroke, and more related efforts are needed to build upon previous programs for stroke prevention, treatment, and management (46). The Chinese government has sponsored a series of largescale regional and nationwide studies to examine the risk factors for stroke and to implement some national programs to promote the prevention and treatment of stroke. China has developed relevant national guidelines accordingly (29,47-50). Since 2015, more than 2,500 hospitals have joined the national, hospital-based, stroke care quality assessment and improvement platform $(51,52)$. However, it seems that the stroke prevention programs implemented in China over the past two decades may not be effective enough at controlling the prevalence of the disease. This is a significant deficit, since caring for stroke survivors has impacts ranging across all aspects of life, from personal to social. It can affect various aspects of the patients' and their family members' well-being, creating physical, mental, and financial stress. Further research and more effective interventions are necessary for addressing these issues. To monitor and optimize these programs, future regional and local stroke surveillance programs, especially for younger age groups, need to be enhanced.

In conclusion, this meta-analysis based on studies published during 2000-2017 showed that stroke prevalence varied significantly across regions, with the highest rate 
in north China and the lowest in southern China. People in the age group of 40-59 old, men, and people living in northern China have the highest prevalence of stroke. China needs to make more vigorous and effective efforts to address the ongoing public health issue of stroke.

\section{Acknowledgments}

Funding: The study was supported in part by research grants from the China Medical Board (Grant No. 16-262), and the National Key Research and Development Program of China (Grant Number: 2017YFC0907200 \& 2017YFC0907201), the National Natural Science Foundation of China (Grant Number: NSFC 81703220).

\section{Footnote}

Reporting Checklist: The authors have completed the PRISMA reporting checklist. Available at http://dx.doi.org/10.21037/ atm-19-4342

Peer Review File: Available at http://dx.doi.org/10.21037/ atm-19-4342

Conflicts of Interest: All authors have completed the ICMJE uniform disclosure form (available at http://dx.doi. org/10.21037/atm-19-4342). The authors have no conflicts of interest to declare.

Ethical Statement: The authors are accountable for all aspects of the work in ensuring that questions related to the accuracy or integrity of any part of the work are appropriately investigated and resolved.

Open Access Statement: This is an Open Access article distributed in accordance with the Creative Commons Attribution-NonCommercial-NoDerivs 4.0 International License (CC BY-NC-ND 4.0), which permits the noncommercial replication and distribution of the article with the strict proviso that no changes or edits are made and the original work is properly cited (including links to both the formal publication through the relevant DOI and the license). See: https://creativecommons.org/licenses/by-nc-nd/4.0/.

\section{References}

1. GBD 2016 Causes of Death Collaborators. Global, regional, and national age-sex specific mortality for
264 causes of death, 1980-2016: a systematic analysis for the Global Burden of Disease Study 2016. Lancet 2017;390:1151-210.

2. Wu S, Wu B, Liu M, et al. Stroke in China: advances and challenges in epidemiology, prevention, and management. Lancet Neurol 2019;18:394-405.

3. Zhou M, Wang H, Zhu J, et al. Cause-specific mortality for 240 causes in China during 1990-2013: a systematic subnational analysis for the Global Burden of Disease Study 2013. Lancet 2016;387:251-72.

4. Suwanwela NC, Poungvarin N. Stroke burden and stroke care system in Asia. Neurol India 2016;64 Suppl: S46-S51.

5. Lozano R, Naghavi M, Foreman K, et al. Global and regional mortality from 235 causes of death for 20 age groups in 1990 and 2010: a systematic analysis for the Global Burden of Disease Study 2010. Lancet 2012;380:2095-128.

6. Murray CJL, Vos T, Lozano R, et al. Disability-adjusted life years (DALYs) for 291 diseases and injuries in 21 regions, 1990-2010: a systematic analysis for the Global Burden of Disease Study 2010. Lancet 2012;380:2197-223.

7. Adeloye D. An estimate of the incidence and prevalence of stroke in Africa: a sys tematic review and meta-analysis. PLoS One 2014;9:e100724.

8. Strong K, Mathers C, Bonita R. Preventing stroke: saving lives around the world. Lancet Neurol 2007;6:182-7.

9. Sun J, Guo Y, Wang X, et al. mHealth For Aging China: Opportunities and Challenges. Aging Dis 2016;7:53-67.

10. Wang W, Jiang B, Sun H, et al. Prevalence, Incidence, and Mortality of Stroke in China: Results from a Nationwide Population-Based Survey of 480687 Adults. Circulation 2017;135:759-71.

11. Hu FB, Liu Y, Willett WC. Preventing chronic diseases by promoting healthy diet and lifestyle: public policy implications for China. Obes Rev 2011;12:552-9.

12. Shamseer L, Moher D, Clarke M, et al. Preferred reporting items for systematic review and meta-analysis protocols (PRISMA-P) 2015: elaboration and explanation. BMJ 2015;350:g7647.

13. Stroup DF, Berlin JA, Morton SC, et al. Meta-analysis of observational studies in epidemiology: a proposal for reporting. Meta-analysis of Observational Studies in Epidemiology (MOOSE) group. JAMA 2000;283:2008-12.

14. Suriah AR, Chong TJ, Yeoh BY. Nutritional situation of a Chinese community. Singapore Med J 1998;39:348-52.

15. Ru X, Wang W, Sun H, et al. Geographical Difference, Rural-urban Transition and Trend in Stroke Prevalence in China: Findings from a National Epidemiological Survey 
of Stroke in China. Sci Rep 2019;9:17330.

16. Li Q, Wu H, Yue W, et al. Prevalence of Stroke and Vascular Risk Factors in China: a Nationwide Communitybased Study. Sci Rep 2017;7:6402.

17. Sun H, Zou X, Liu L. Epidemiological factors of stroke: a survey of the current status in china. J Stroke 2013;15:109-14.

18. Reynolds K, Gu D, Muntner P, et al. Geographic variations in the prevalence, awareness, treatment and control of hypertension in China. J Hypertens 2003;21:1273-81.

19. Wu Y, Huxley R, Li L, et al. Prevalence, awareness, treatment, and control of hypertension in China: data from the China National Nutrition and Health Survey 2002. Circulation 2008;118:2679-86.

20. Xu G, Ma M, Liu X, et al. Is there a stroke belt in China and why? Stroke 2013;44:1775-83.

21. Bonita R, Solomon N, Broad JB. Prevalence of stroke and stroke-related disability. Estimates from the Auckland stroke studies. Stroke 1997;28:1898-902.

22. Shi Z, Taylor AW, Hu G, et al. Rice intake, weight change and risk of the metabolic syndrome development among Chinese adults: the Jiangsu Nutrition Study (JIN). Asia Pac J Clin Nutr 2012;21:35-43.

23. Ferri CP, Schoenborn C, Kalra L, et al. Prevalence of stroke and related burden among older people living in Latin America, India and China. J Neurol Neurosurg Psychiatry 2011;82:1074-82.

24. Feigin VL, Lawes CMM, Bennett DA, et al. Stroke epidemiology: a review of population-based studies of incidence, prevalence, and case-fatality in the late 20th century. Lancet Neurol 2003;2:43-53.

25. Gan Y, Wu J, Zhang S, et al. Prevalence and risk factors associated with stroke in middle-aged and older Chinese: A community-based cross-sectional study. Sci Rep 2017;7:9501.

26. Zhang FL, Guo ZN, Wu YH, et al. Prevalence of stroke and associated risk factors: a population based cross sectional study from northeast China. BMJ Open 2017;7:e015758.

27. Zhao Y, Yan H, Marshall RJ, et al. Trends in population blood pressure and prevalence, awareness, treatment, and control of hypertension among middle-aged and older adults in a rural area of Northwest China from 1982 to 2010. PLoS One 2013;8:e61779.

28. Lu J, Lu Y, Wang X, et al. Prevalence, awareness, treatment, and control of hypertension in China: data from 1.7 million adults in a population-based screening study (China PEACE Million Persons Project). Lancet
2017;390:2549-58.

29. Guzik A, Bushnell C. Stroke Epidemiology and Risk Factor Management. Continuum 2017;23:15-39.

30. Wang Y, Wang X, Kong Y, et al. The Great Chinese Famine leads to shorter and overweight females in Chongqing Chinese population after 50 years. Obesity 2010;18:588-92.

31. Wang J, Li Y, Han X, et al. Exposure to the Chinese Famine in Childhood Increases Type 2 Diabetes Risk in Adults. J Nutr 2016;146:2289-95.

32. Shi Z, Nicholls SJ, Taylor AW, et al. Early life exposure to Chinese famine modifies the association between hypertension and cardiovascular disease. J Hypertens 2018;36:54-60.

33. Fransen HP, Peeters PHM, Beulens JWJ, et al. Exposure to Famine at a Young Age and Unhealthy Lifestyle Behavior Later in Life. PLoS One 2016;11:e0156609.

34. Appelros P, Stegmayr B, Terént A. Sex differences in stroke epidemiology: a systematic review. Stroke 2009;40:1082-90.

35. Venketasubramanian N, Tan LCS, Sahadevan S, et al. Prevalence of stroke among Chinese, Malay, and Indian Singaporeans: a community-based tri-racial cross-sectional survey. Stroke 2005;36:551-6.

36. Zhang C, Lan T, Zhe Y, et al. Epidemiology Investigation of stroke among Mongolian and Han population aged over 45 in Inner Mongolia. Sci Rep 2017;7:45710.

37. Liu S, Zhang M, Yang L, et al. Prevalence and patterns of tobacco smoking among Chinese adult men and women: findings of the 2010 national smoking survey. J Epidemiol Community Health 2017;71:154-61.

38. Millwood IY, Li L, Smith M, et al. Alcohol consumption in 0.5 million people from 10 diverse regions of China: prevalence, patterns and socio-demographic and healthrelated correlates. Int J Epidemiol 2017;46:2103.

39. Jackson G. Tobacco: the most important preventable cause of cardiovascular disease. Int J Clin Pract 2010;64:279-80.

40. O'Donnell M, Yusuf S. Tackling the global burden of stroke: the need for large-scale international studies. Lancet Neurol 2009;8:306-7.

41. Lemon SC, Rosal MC, Zapka J, et al. Contributions of weight perceptions to weight loss attempts: differences by body mass index and gender. Body Image 2009;6:90-6.

42. Chen Y, Peng Q, Yang Y, et al. The prevalence and increasing trends of overweight, general obesity, and abdominal obesity among Chinese adults: a repeated crosssectional study. BMC Public Health 2019;19:1293.

43. Adeboye B, Bermano G, Rolland C. Obesity and its health 
impact in Africa: a systematic review. Cardiovasc J Afr 2012;23:512-21.

44. Alonso de Leciñana M, Egido JA, Fernández C, et al. Risk of ischemic stroke and lifetime estrogen exposure. Neurology 2007;68:33-8.

45. Ritzel RM, Capozzi LA, McCullough LD. Sex, stroke, and inflammation: the potential for estrogenmediated immunoprotection in stroke. Horm Behav 2013;63:238-53.

46. Li Z, Jiang Y, Li H, et al. China's response to the rising stroke burden. BMJ 2019;364:1879.

47. Guan T, Ma J, Li M, et al. Rapid transitions in the epidemiology of stroke and its risk factors in China from 2002 to 2013. Neurology 2017;89:53-61.

48. Zhang J, Astell-Burt T, Seo DC, et al. Multilevel evaluation of 'China Healthy Lifestyles for All', a nationwide initiative to promote lower intakes of salt and

Cite this article as: Liu J, Shi Z, Bai R, Zheng J, Ma S, Wei J, Liu G, Wang Y. Temporal, geographical and demographic trends of stroke prevalence in China: a systematic review and meta-analysis. Ann Transl Med 2020;8(21):1432. doi: 10.21037/atm-19-4342 edible oil. Prev Med 2014;67:210-5.

49. Li Z, Wang C, Zhao X, et al. Substantial Progress Yet Significant Opportunity for Improvement in Stroke Care in China. Stroke 2016;47:2843-9.

50. Wang Y, Li Z, Zhao X, et al. Stroke care quality in China: Substantial improvement, and a huge challenge and opportunity. Int J Stroke 2017;12:229-35.

51. Wang Y, Li Z, Xian Y, et al. Rationale and design of a cluster-randomized multifaceted intervention trial to improve stroke care quality in China: The GOLDEN BRIDGE-Acute Ischemic Stroke. Am Heart J 2015;169:767-774.e2.

52. Wang Y, Li Z, Wang Y, et al. Chinese Stroke Center Alliance: a national effort to improve healthcare quality for acute stroke and transient ischaemic attack: rationale, design and preliminary findings. Stroke Vasc Neurol 2018;3:256-62. 